PROCEEDINGS OF THE

AMERICAN MATHEMATICAL SOCIETY

Volume 130, Number 1, Pages 121-127

S 0002-9939(01)06154-8

Article electronically published on June 8, 2001

\title{
ON THE SPLIT PROPERTY FOR INCLUSIONS OF $W^{*}$-ALGEBRAS
}

\author{
FRANCESCO FIDALEO
}

(Communicated by David R. Larson)

\begin{abstract}
A characterization of the quasi-split property for an inclusion of $W^{*}$-algebras in terms of the metrically nuclear maps is established. This result extends the known characterization relative to inclusions of $W^{*}$-factors. An application to type $I$ von Neumann algebras is also presented.
\end{abstract}

\section{INTRODUCTION}

The split property for inclusions of von Neumann algebras is of particular interest from a theoretical viewpoint although it was first introduced and intensively studied for the various applications in Quantum Field Theory. For the physical applications, the relation of the split property with several nuclearity conditions is also of interest; see [3], and [14 for a review.

To make the connection between the split property and the various nuclearity conditions more transparent, canonical non-commutative embeddings $M \hookrightarrow$ $L^{p}(M), p=1,2$, were first considered in [2]. Namely, given an inclusion $N \subset M$ of von Neumann algebras with $M$ acting standardly on the Hilbert space $\mathcal{H}$ with a cyclic and separating vector $\Omega$, one can consider the following embeddings, canonically constructed via the modular operators $\Delta, J$ associated to $\Omega$ :

$$
\begin{gathered}
\Phi_{1}: a \in M \rightarrow\langle\cdot \Omega \mid J a \Omega\rangle \in M_{*}, \\
\Phi_{2}: a \in M \rightarrow \Delta^{1 / 4} a \Omega \in \mathcal{H} .
\end{gathered}
$$

The main result of [2] is the following. Let $N \subset M$ be a factor-subfactor inclusion. Then the nuclearity condition for the restriction to $N$ of any of the above maps ensures the split property for the inclusion. Conversely, if $N \subset M$ is a split inclusion, the above embeddings, when restricted to $N$, are nuclear for a dense set of cyclic separating vectors for $M$.

Unfortunately, the nuclearity condition is a stronger condition than the split property when some privileged state (as e.g. the vacuum state for the physical applications) is kept fixed. Hence a complete characterization of the split property in terms of properties of the $L^{p}$ embeddings would be desirable. This approach has

Received by the editors June 8, 2000.

2000 Mathematics Subject Classification. Primary 46L37; Secondary 46L07, 46L10.

Key words and phrases. Classifications, factors, linear spaces of operators.

(C)2001 American Mathematical Society 
been followed in $\left[8\right.$ in order to solve the question for the $L^{1}$ embedding. Namely, given an inclusion $N \subset M$ of von Neumann algebras and the $L^{1}$ embedding $\Phi_{1}$, constructed as in (1) by any standard vector $\Omega$ for $M$, it has been shown that the metrically nuclear condition for $\Phi_{1\lceil N}$ characterizes in a complete way the split property for the inclusion. Moreover, some conditions (weaker than nuclearity) which ensure the split property were established in [9] relative to the $L^{2}$ embedding. All these results were obtained for inclusions of von Neumann factors. Hence it could be interesting to extend some of the above results to the general case of inclusions of von Neumann algebras. This is of certain interest also for the physical applications where the local algebras of observables could have a non-trivial centre; see [1].

In the present note, following the strategy of [8], we characterize the quasisplit property for an inclusion of von Neumann algebras with (possibly) non-trivial centres in terms of the metrically nuclear maps. An application to the structure of type $I$ von Neumann algebras is also presented.

The key point for the characterization of the (quasi-)split property is the new theory of operator spaces and some classes of completely bounded maps between them. For the theory of operator spaces we refer the reader to [6, 11, 12] and to the literature cited therein.

We also refer the reader to [7, 8 for the definition and the basic properties of the metrically nuclear maps $\mathfrak{N}(E, F)$ between the operator spaces $E, F[$

Finally, we recall that an inclusion $N \subset M$ of von Neumann algebras is said to be split if there exists an interpolating type $I$ von Neumann factor $F$, that is, $N \subset F \subset M$. The last condition implies (and in many interesting situations is equivalent to) the existence of a normal *-isomorphism between $N \bar{\otimes} M^{\circ}$ and $N \vee M^{\prime}$ extending $a \otimes b \rightarrow a J b^{*} J$, after representing $M$ in standard form; see [5].

\section{The QUASI-SPLIT PROPERTY FOR INCLUSIONS OF $W^{*}$-ALGEBRAS}

This section follows Section 1 of 2 where the split property for inclusions of factors is related to the extendibility of the canonical non-commutative embeddings $\Phi_{i}, i=1,2$. Here we deal with the general case of inclusions of von Neumann algebras. This framework also covers the commutative case which has been treated in some detail in 22. We note that the subject contained in this section has a natural description in terms of Connes correspondence (4). Accordingly, we adopt the correspondence formalism in the sequel.

We start with an inclusion $N \subset M$ of von Neumann algebras always with separable preduals. Taking a normal faithful state $\omega \in M_{*}$ and representing $M$ on the Hilbert space $\mathcal{H} \equiv L^{2}(M)$ by the GNS representation relative to $\omega$, we consider the Tomita operators $J, \Delta$ relative to it. By $M^{\circ}$ we denote the opposite algebra of $M$.

Definition 1. The inclusion $N \subset M$ is said to be quasi-split if the map

$$
a \otimes b \in N \otimes M^{\circ} \rightarrow a J b^{*} J \in \mathcal{B}\left(L^{2}(M)\right)
$$

extends to a normal $*$-homomorphism $\eta$ of $N \bar{\otimes} M^{\circ}$ onto $N \vee M^{\prime}$.

We remark that, since the standard representation is unique up to unitary equivalence, the quasi-split property is really an intrinsic property of the inclusion.

\footnotetext{
${ }^{1}$ In [8] the metrically nuclear maps, denoted as $\mathcal{D}(E, F)$, are called the matrix-decomposable maps.
} 
Let $N, M$ be von Neumann algebras; a $N-M$ correspondence is a separable Hilbert space $\mathcal{H}$ which is also a $N-M$ bimodule with (commuting) normal left and right actions of $N, M$ respectively.

The standard representation of $M$ gives rise to a correspondence which is unique up to unitary equivalence, just the identity $M-M$ correspondence ${ }_{M} \operatorname{id}_{M}$ on $L^{2}(M)$ determined by the map

$$
a \otimes b \in M \otimes M^{\circ} \rightarrow a J b^{*} J \in \mathcal{B}\left(L^{2}(M)\right)
$$

If we have an inclusion $N \subset M$ of von Neumann algebras, we obtain a (uniquely determined) $N-M$ correspondence if we restrict ${ }_{M} \operatorname{id}_{M}$ to $N$ on the left. We indicate it by $N \operatorname{id}_{M}$.

Definition 2. An $N-M$ correspondence $\sigma$ is said to be split if there exist normal faithful representations $\pi, \pi^{\circ}$ of $N, M^{\circ}$ on separable Hilbert spaces $\mathcal{H}_{\pi}, \mathcal{H}_{\pi^{\circ}}$ respectively, such that $\sigma$ is unitarily equivalent to $\pi \otimes \pi^{\circ}\left(\sigma \cong \pi \otimes \pi^{\circ}\right.$ for short).

The term coarse is also used when $\pi, \pi^{\circ}$ are the standard representations of $N$, $M^{\circ}$ respectively.

Now we show that the quasi-split property can be viewed as a property of ${ }_{N} \mathrm{id} M$.

Proposition 1. Let $N \subset M$ be an inclusion of von Neumann algebras with separable preduals.

The following statements are equivalent:

(i) $N \subset M$ is a quasi-split inclusion;

(ii) ${ }_{N} \operatorname{id}_{M} \prec \sigma$, where $\sigma$ is a $N-M$ split correspondence and $\prec$ means the containment of representations.

Proof. (i) $\Rightarrow$ (ii) If the map (3) extends to a normal $*$-homomorphism $\eta$ of $N \bar{\otimes} M^{\circ}$ onto $N \bigvee M^{\prime}$, then $\eta=\eta_{1} \circ \eta_{2} \circ \eta_{3}$ where $\eta_{3}$ is an amplification, $\eta_{2}$ an induction and $\eta_{1}$ a spatial isomorphism; see [13. Hence

$$
\eta(a \otimes b)=v^{*}(a \otimes b \otimes I) v
$$

where $v: L^{2}(M) \rightarrow L^{2}(M) \otimes L^{2}(M) \otimes \mathcal{H}$ is an isometry. Namely, the $N-M$ correspondence ${ }_{N} \mathrm{id}_{M}$ is contained in a split one.

(ii) $\Rightarrow$ (i) If ${ }_{N} \mathrm{id}_{M}$ is a subcorrespondence of a split one, we have a subrepresentation of the normal representation $\pi \otimes \pi^{\circ}$ of $N \bar{\otimes} M^{\circ}$; see [15], Theorem IV.5.2. Hence ${ }_{N} \operatorname{id}_{M}$ uniquely defines a normal representation of $N \bar{\otimes} M^{\circ}$ that is a normal map $\eta$ of $N \bar{\otimes} M^{\circ}$ onto $N \vee M^{\prime}$ which extends (3).

When a faithful state $\omega \in M_{*}$ is kept fixed, one can construct the non-commutative embeddings $\Phi_{1}, \Phi_{2}$ given in (1), (2). Then, following [2], one can study the extendibility properties of $\Phi_{i}, i=1,2$.

A completely positive normal map $\Phi: N \rightarrow L^{p}(M), 1 \leq p<+\infty$, is said to be extendible (according with [2], page 236) if, whenever $\widetilde{N} \supset N$ is another von Neumann algebra with separable predual, there exists a completely positive normal 
map $\widetilde{\Phi}$ which yields the following commutative diagram:

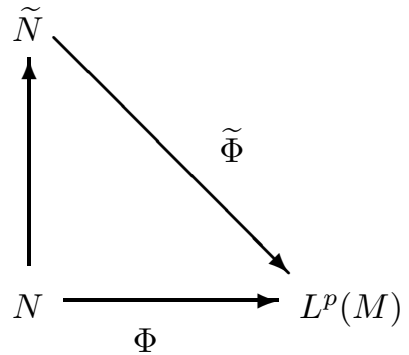

Here complete positivity has an obvious meaning if one considers for $n \in \mathbb{N}$ the hierarchy of positive cones $L^{p}\left(\mathbb{M}_{n}(M)\right)_{+} \subset L^{p}\left(\mathbb{M}_{n}(M)\right)$; see [10].

The following proposition is the natural generalization of [2], Proposition 1.1.

Proposition 2. Let $N \subset M$ be an inclusion of von Neumann algebras with separable preduals, and $\omega \in M_{*}$ a faithful state. Consider the embeddings $\Phi_{i}, i=1,2$, relative to $\omega$.

The following statements are equivalent:

(i) $N \subset M$ is a quasi-split inclusion;

(ii) $\Phi_{1\lceil N}: N \rightarrow L^{1}(M)$ is extendible;

(iii) $\Phi_{2\lceil N}: N \rightarrow L^{2}(M)$ is extendible.

Proof. (iii) $\Rightarrow$ (ii) We can consider the commutative diagram

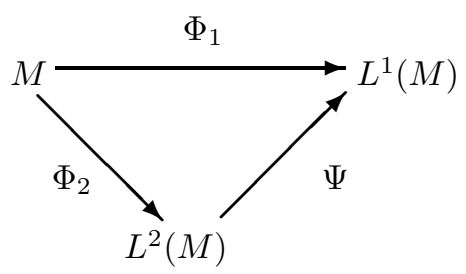

where $\Psi$, given by

$$
\Psi(x):=\left\langle\Delta^{1 / 4} \cdot \Omega \mid J x\right\rangle,
$$

is computed by the transpose map of $\Phi_{2}$. So if $\Phi_{2\lceil N}$ is extendible, then $\Phi_{1\lceil N}$ is extendible as well.

(ii) $\Rightarrow$ (i) Let $F$ be a type $I$ factor with separable predual containing $N$ and $\widetilde{\Phi}_{1}$ the corresponding completely positive normal extension of $\Phi_{1}$. Then

$$
\widetilde{\varphi}(f \otimes b):=\widetilde{\Phi}_{1}(f)(b)
$$

gives rise to an $F-M$ correspondence $\left(\sigma, \mathcal{H}_{\sigma}\right)$; see [15], Proposition IV.4.6. Moreover, the $F-M$ correspondence $\left(\sigma, \mathcal{H}_{\sigma}\right)$, when restricted to $N$ on the left, contains ${ }_{N} \operatorname{id}_{M}$. The assertion now follows as the standard representation of $M$ is faithful. 
(i) $\Rightarrow$ (iii) It is enough to show that $\Phi_{2}$ extends to a type $I$ factor with separable predual containing $N$ as a unital subalgebra. Let $\left(\pi \otimes \pi^{\circ}, \mathcal{H}_{\pi} \otimes \mathcal{H}_{\pi^{\circ}}\right)$ be a split correspondence containing ${ }_{N} \mathrm{id}_{M}$. Then there exists an isometry $v: L^{2}(M) \rightarrow$ $\mathcal{H}_{\pi} \otimes \mathcal{H}_{\pi}$. with range projection $v v^{*} \in \pi(N)^{\prime} \bar{\otimes} \pi^{\circ}\left(M^{\circ}\right)^{\prime}$, such that, for $a \in N$, $b \in M^{\circ}$, we get

$$
a J b^{*} J=v^{*} \pi(a) \otimes \pi^{\circ}(b) v .
$$

As $v v^{*} \in \mathcal{B}\left(\mathcal{H}_{\pi}\right) \bar{\otimes} \pi^{\circ}\left(M^{\circ}\right)^{\prime}$, it is easy to show that $v^{*}\left(\mathcal{B}\left(\mathcal{H}_{\pi}\right) \otimes I\right) v \subset M$. Now we define $\widetilde{\Phi}_{2}: \mathcal{B}\left(\mathcal{H}_{\pi}\right) \rightarrow L^{2}(M)$ as

$$
\widetilde{\Phi}_{2}(f):=\Delta^{1 / 4} v^{*}(f \otimes I) v \Omega
$$

which is a completely positive normal extension of $\Phi_{2}$ to the type $I$ factor $\mathcal{B}\left(\mathcal{H}_{\pi}\right)$. The general case is recovered by taking the tensor product $F_{\infty} \bar{\otimes} \mathcal{B}\left(\mathcal{H}_{\pi}\right)$ with the hyperfinite type $I$ factor $F_{\infty}$ and consider any normal conditional expectation $\epsilon$ : $F_{\infty} \bar{\otimes} \mathcal{B}\left(\mathcal{H}_{\pi}\right) \rightarrow \mathcal{B}\left(\mathcal{H}_{\pi}\right)$.

We note that Proposition 1.1 of [2] can be immediately recovered as a corollary of the above result.

Proposition 3. If $N \subset M$ is an inclusion of von Neumann factors with separable preduals, then the condition (i) in Proposition 2 can be replaced by the a-priori stronger condition

(i') $N \subset M$ is a split inclusion.

Proof. Let $\eta$ be the normal map which extends (3) to all of $N \bar{\otimes} M^{\circ}$. If $N$ and $M$ are both factors, then $\operatorname{ker} \eta=\{0\}$, that is, $\eta$ is a normal $*$-isomorphism onto $N \vee M^{\prime}$. The proof follows as the last condition turns out to be equivalent to the split property; see [5], Corollary 1.

\section{Metrically NuClear MAPs AND The QUASI-SPlit PROPERTY}

In the present section we show that the quasi-split property for an inclusion $N \subset$ $M$ of von Neumann algebras is characterized by the metrically nuclear condition for the $L^{1}$ embedding $\Phi_{1}$ of $N$ into the predual of $M$. Such a characterization parallels the analogous one given in 8] for factor-subfactor inclusions. It can be naturally applied to inclusions arising from Quantum Field Theory, where the local algebras could be type $I I I$ algebras with a non-trivial centre 2

Theorem 1. Let $N \subset M$ be an inclusion of von Neumann algebras with separable preduals, and $\omega \in M_{*}$ a faithful state. Consider the $L^{1}$ embedding $\Phi_{1}$ relative to $\omega$.

The following statements are equivalent:

(i) $N \subset M$ is a quasi-split inclusion;

(ii) $\Phi_{1\lceil N} \in \mathfrak{N}\left(N, M^{\circ}\right)$.

Proof. (i) $\Rightarrow$ (ii) Consider on $N \vee M^{\prime}$ the vector state $\varphi(c):=\langle c \Omega \mid \Omega\rangle$. As $N \subset M$ is quasi-split, there exists a normal $*$-homomorphism $\eta$ of $N \bar{\otimes} M^{\circ}$ onto $N \vee M^{\prime}$ extending the algebraic homomorphism (3). Accordingly, the composite map $\varphi \circ \eta$ gives rise to a normal state on $N \bar{\otimes} M^{\circ}$, that is, $\varphi \circ \eta \in N_{*} \widehat{\otimes} M_{*}^{\circ}$; see [6], Theorem 3.2. As $\Phi_{1}(a)(b)=\varphi \circ \eta(a \otimes b)$, the proof becomes complete taking into account Definition 2.1 of [8] relative to the metrically nuclear maps.

\footnotetext{
${ }^{2}$ The proof of Corollary 1 of [9], being incomplete relative to the implication (iii) $\Rightarrow$ (iv), can be completed taking into account Theorem[1]
} 
(ii) $\Rightarrow$ (i) If $\Phi_{1\lceil N} \in \mathfrak{N}\left(N, M^{\circ}{ }^{*}\right)$, then $\Phi_{1}(a)(b)=: \varphi(a \otimes b)$ defines a normal state on all of $N \bar{\otimes} M^{\circ}$ by [8], Lemma 3.2. Now let $\widetilde{N} \supset N$ be a von Neumann algebra with separable predual containing $N$ and consider a normal state $\widetilde{\varphi}$ on $\widetilde{N} \bar{\otimes} M^{\circ}$ extending $\varphi$. Then $\widetilde{\Phi}_{1}(a):=\widetilde{\varphi}(a \otimes \cdot)$ is a completely positive normal map extending $\Phi_{1}$. The proof follows by Proposition 2 .

Remark 1 . We note that, by the above considerations, Theorem 2 in [9] can be easily generalized to inclusions of von Neumann algebras replacing the split condition with the quasi-split one. We leave the details to the reader.

Finally, following analogous characterizations contained in [2], Corollary 2.9, and in [9, Proposition 7, we apply Theorem 1 to the structure of type $I$ von Neumann algebras.

Theorem 2. Let $M$ be a von Neumann algebra with separable predual, and $\omega \in M_{*}$ a faithful state. Consider the $L^{1}$ embedding $\Phi_{1}$ relative to $\omega$.

The following statements are equivalent:

(i) $M$ is atomic;

(ii) $\Phi_{1} \in \mathfrak{N}\left(M, M^{\circ}{ }_{*}\right)$.

Proof. (i) $\Rightarrow$ (ii) As $M$ is atomic, it is a direct sum of type $I$ factors $M_{i}$. Let $\left\{p_{i}\right\}_{i \in \mathbf{n}}$ be the set of the minimal central projections of $M$, where $\mathbf{n}$ is any finite or countable set describing the dimension of $\mathrm{Z}(M)$. In such a way $M_{i}=M_{p_{i}}$. Consider the ${ }^{*-}$ isomorphisms $\eta_{i}: M_{i} \bar{\otimes} M_{i}^{\circ} \rightarrow M_{i} \vee M_{i}^{\prime}$ extending $a p_{i} \otimes b p_{i} \rightarrow a J b^{*} J p_{i}$. These isomorphisms always exist as $M_{i}$ are all type $I$ factors. Then it is easy to verify that

$$
\eta(c):=\bigoplus_{i \in \mathbf{n}} \eta_{i}\left(c p_{i} \otimes p_{i}\right)
$$

is a normal $*$-homomorpism of $M \bar{\otimes} M^{\circ}$ onto $M \vee M^{\prime}$ which extends the map $a \otimes b \rightarrow$ $a J b^{*} J$. Namely, the trivial inclusion based on $M$ is quasi-split, that is, $\Phi_{1} \in$ $\mathfrak{N}\left(M, M^{\circ}{ }_{*}\right)$ by Theorem 1.

(ii) $\Rightarrow$ (i) If (ii) is satisfied, then the inclusion based on $M$ is quasi-split again by Theorem 1 Namely, there exists a normal $*$-homomorphism $\eta$ of $M \bar{\otimes} M^{\circ}$ onto $M \vee M^{\prime}$ which extends the map $a \otimes b \rightarrow a J b^{*} J$.

Consider first the centre of $M$. We start by noticing that $\mathrm{Z}(M)$ is isomorphic to $L^{\infty}(X, \mu)$ whereas $\mathrm{Z}(M) \bar{\otimes} \mathrm{Z}(M)$ is isomorphic to $L^{\infty}(X \times X, \mu \times \mu)$ where $(X, \mu)$ is a standard measure space; see [15], Theorem III.1.22. In such a situation, the map $a \otimes b \rightarrow a J b^{*} J$ leads to

$$
a(x) b(y) \in L^{\infty}(X \times X, \mu \times \mu) \rightarrow a(x) b(x) \in L^{\infty}(X, \mu)
$$

when it is restricted to $\mathrm{Z}(M) \bar{\otimes} \mathrm{Z}(M)$. But, it is straightforward to verify that the above map is indeed a normal $*$-homomorphism if and only if $(X, \mu)$ is purely atomic. Then $\mathrm{Z}(M)$ is atomic.

Now let $M_{i}$ be the factors appearing in the decomposition of $M$. Restricting the map $\eta$ to $M_{i} \bar{\otimes} M_{i}^{\circ}$, we conclude that the trivial inclusions based on the factors $M_{i}$ are all quasi-split, hence split by Proposition 3 Then the $M_{i}$ are all type $I$ factors. Namely, $M$ is a direct sum of type $I$ factors, that is, $M$ is atomic. 


\section{REFERENCES}

[1] Buchholz D., D'Antoni C., Fredenhagen K. The universal structures of local algebras, Commun. Math. Phys. 111 (1987), 123-135. MR 88j:46055

[2] Buchholz D., D'Antoni C., Longo, R. Nuclear maps and modular structures I, J. Funct. Anal. 88 (1990), 233-250. MR 91f:46080

[3] Buchholz D., D'Antoni C., Longo R. Nuclear maps and modular structures II, Commun. Math. Phys. 129 (1990), 115-138. MR 91k:81081

[4] Connes A., Jones V. F. R. Property $T$ for von Neumann algebras, Bull. London Math. Soc. 17 (1985), 57-62. MR 86a:46083

[5] D'Antoni C., Longo R. Interpolation by type I factors and the flip automorphism, J. Funct. Anal. 51 (1983), 361-371. MR 84k:46047

[6] Effros E., Ruan Z.-J. On approximation properties for operator spaces, International J. Math. 1 (1990), 163-187. MR 92g:46089

[7] Effros E., Ruan Z.-J. Mapping spaces and liftings of operator spaces, Proc. London Math. Soc. 69 (1994), 171-197. MR 96c:46074a

[8] Fidaleo F. Operator space structures and the split property, J. Operator Theory 31 (1994), 207-218. MR 97a:46069

[9] Fidaleo F. Some operator ideals in non-commutative functional analysis, Z. Anal. Anwendungen 17 (1998), 759-776. MR 99i:46041

[10] Haagerup U. $L^{p}$-spaces associated with an arbitrary von Neumann algebra, Colloques internationaux CNRS 274 (1979) 175-184. MR 81e:46050

[11] Paulsen V. I. Completely bounded maps and dilations, Longman Scientific \& Technical (1986). MR 88h:46111

[12] Ruan Z.-J., Subspaces of $C^{*}$-algebras, J. Funct. Anal. 76 (1988), 217-230. MR 89h:46082

[13] Stratila S., Zsido L. Lectures on von Neumann algebras, Abacus Press (1979). MR 81j:46089

[14] Summers S. J. On the independence of local algebras in Quantum Field Theory, Rev. Math. Phys. 2 (1990), 201-247. MR 92c:81086

[15] Takesaki M. Theory of operator algebras I, Springer (1979). MR 81e:46038

Dipartimento di Matematica and Centro Interdisciplinare Vito Volterra, II UniverSità di Roma "Tor Vergata", Via della Ricerca Scientifica, 00133 Roma, Italy

E-mail address: fidaleo@axp.mat.uniroma2.it 